\title{
Brazil's Initial Experience in Widening Awareness of Familial Hypercholesterolemia with Patients Associations
}

\author{
Anita LR Saldanha ${ }^{1}$, Patrícia Vieira de Luca ${ }^{1}$, Maria Cristina de Oliveira Izar ${ }^{2}$, Tania Leme da Rocha \\ Martinez ${ }^{1 *}$ \\ ${ }^{1}$ Nephrology Department, BP - A Beneficência Portuguesa de São Paulo, Brazil \\ ${ }^{2}$ Lipid Department - Federal University of São Paulo - UNIFESP, Brazil
}

*Corresponding author: Tania Leme da Rocha Martinez, Nephrology Department, BP - A Beneficência Portuguesa de São Paulo, São Paulo, Brazil.
Received Date: October 13, 2021

Published Date: November 12, 2021

\begin{abstract}
In this paper we describe the steps taken for broadening the publical, midiatic and all heart professional awareness in Brazil of Familial Hypercholesterolemia and the cardiovascular risk it carries, carries encompassing the strategic plan, advocacy, its purpose, how the Brazilian Familial Hypercholesterolemia Patients Association was born, this mission, objectives, and the reality of familial hypercholesterolemia patients in Brazil.
\end{abstract}

Keywords: Familial hypercholesterolemia; Patients associations; Midia; Public awareness; Advocacy; Stakeholders

Abbreviations: FH: Family Hypercholesterolemia; FHPA: Familial Hypercholesterolemia Patients Association

\section{Opinion}

In this paper we describe the steps taken for broadening the publical, midiatic and all heart professional awareness in Brazil of Familial Hypercholesterolemia and the cardiovascular risk it carries.

\section{Strategic Plan}

\section{Strategic objectives}

1. Map people with suspected or diagnosed family hypercholesterolemia (FH).

2. Increase and engage the number of members: patients, family members and health professionals towards the Association.

3. Expand the knowledge of patients, health professionals, government agencies and society in general about the disease, its diagnosis and treatment.
4. Promote the recognition of the disease with the creation of specific ICD (International Classification of Diseases).

5. Support the development of scientific research for the treatment of $\mathrm{FH}$ and related diseases.

6. Influence entities, managers and politicians to guarantee patients' rights.

\section{Advocacy}

It consists of a set of actions that aim to influence the formulation, approval and implementation of public policies with the Legislative, Executive and Judiciary branches and society, through networking and midia mobilization. This is a topic of interest for third sector organizations and researchers who aim to bring about changes in society from public policies in the areas of health, environment, human rights, children and adolescents, education, consumer law, work, among others. 


\section{What is an Association? What is its purpose?}

\section{How was the Brazilian Familial Hypercholesterolemia Patients Association born (FHPA)?}

FHPA is a non-profit entity, founded by patients with $\mathrm{FH}$ conditions, which has a scientific council with the support of actions and decisions of the Association.

\section{What is the FHPA mission?}

Stakeholders' awareness about FH and promote equal access to diagnosis and treatment, making an alliance with all involved to improve disease rates.

\section{Main objectives}

a. Promote contact between individuals, family groups and associations seeking information about FH and other geneticbased dyslipidemias in Brazil and other countries.

b. Encourage, promote, and support alliances with national and international groups that work in the support and development of scientific research and treatments for $\mathrm{FH}$ patients.

c. Support and disseminate scientific research for the treatment and cure of FH-related diseases, disseminating data and medical information on treatment and cure to patients, family members, guardians, and other stakeholders.

d. Promote social and legal assistance to FH sufferers and their families or guardians, providing them with the guidance and defense they need.

e. Encourage and promote extensive knowledge about FH by conducting courses, conferences, lectures and distribution of books, videos, periodicals, brochures, and all available media.

f. Support programs to improve the medical area by conducting courses, congresses, conferences, and lectures.

g. Encourage volunteering by training support groups for FH patients in the national territory and in other countries.

\section{The reality of FH patients in Brazil}

Challenges faced by the patient:

I. Lack of information

II. Ignorance of the medical class

III. No referral for second medical opinion

IV. Restricted access to genetic diagnoses

V. Late diagnosis

VI. Lack of knowledge and lack of access to adequate treatment

VII. Lack of maintenance and follow-up of the disease

VIII. Lack of psychological support for patients and their families

IX. Lack of culture and difficulty in accessing healthy eating

\section{FHPA Strategic Guidelines}

\section{Vision of the future}

Mission: raise society's awareness about FH and promote equal access to diagnosis and treatment, making an alliance with all involved to improve disease rates.

Essence of the mission: raise awareness, promote access, make alliances.

Vision of the future: a country where people with FH are diagnosed early, treated appropriately, and do not die because of the disease.

\section{Acknowledgment}

To the Familial Hypercholesterolemia Foundation (FHF) for its example and support along the way, to the International Atherosclerosis Society (IAS) and the European Atherosclerosis Society (EAS)

\section{Conflicts of Interest}

No conflict of interest. 